\title{
Flux transfer events on the high-latitude magnetopause: Interball-1 observations
}

\author{
D. G. Sibeck ${ }^{1}$, G. I. Korotova ${ }^{2}$, V. Petrov ${ }^{2}$, V. Styazhkin ${ }^{2}$, and T. J. Rosenberg ${ }^{3}$ \\ ${ }^{1}$ Code 612.3, NASA/GSFC, Greenbelt, MD 20771, USA \\ ${ }^{2}$ IZMIRAN, Troitsk, Moscow Region, 142190, Russia \\ ${ }^{3}$ Institute for Physical Science and Technology, University of Maryland, College Park, MD 20742, USA
}

Received: 10 August 2005 - Revised: 9 November 2005 - Accepted: 10 November 2005 - Published: 21 December 2005

\begin{abstract}
We present case and statistical studies of flux transfer events (FTEs) observed by Interball-1 on the highlatitude magnetopause. The case studies provide observations of FTEs in the cusp during periods of southward interplanetary magnetic field (IMF) orientation and on the magnetopause poleward of the cusp during periods of strongly northward IMF orientation. We interpret the former in terms of reconnection on the equatorial magnetopause and subsequent antisunward motion of FTEs into the cusps. We interpret the latter in terms of bursty antiparallel merging on the high-latitude magnetopause. A statistical survey demonstrates that events observed equatorward of the cusp show a marked tendency to occur for antiparallel (northward) magnetospheric and (southward) magnetosheath magnetic field orientations, whereas events observed poleward of the cusps tend to occur for either strongly parallel or antiparallel configurations. We suggest that this discrepancy implies that events observed poleward of the cusps originate both locally and on the equatorial magnetopause. Finally, we use the sense of the bipolar signature and the prevailing magnetic field orientation to demonstrate that almost all events move antisunward, i.e. that at these latitudes pressure gradients determine the motion of FTEs and not magnetic curvature forces.
\end{abstract}

Keywords. Magnetospheric physics (Magnetopause, cusp and boundary layers; Solar wind-magnetosphere interactions)

\section{Introduction}

Theory and numerical simulations predict the characteristics of transient events produced by bursty merging on the magnetopause. Simultaneous bursty merging along $\mathrm{n}$ extended merging lines generates a pair of bubbles and $n-1$ flux ropes (Lee and Fu, 1985; Southwood et al., 1988; Ku and Sibeck,

Correspondence to: D. G. Sibeck

(david.g.sibeck@nasa.gov)
2000). The dimensions of the bubbles and flux ropes of interconnected magnetosheath and magnetospheric magnetic field lines grow with distance and time as they move away from the reconnection site(s) at velocities determined by the balance of pressure gradient and magnetic curvature forces (Cowley and Owen, 1989). Events generated by currentdriven instabilities should be most common on the dayside low- and mid-latitude magnetopause during periods of southward IMF orientation.

Bubbles and flux ropes moving along the magnetopause displace the surrounding media and generate characteristic magnetic field and plasma signatures (Cowley, 1982). As illustrated in Fig. 1a, northward-moving bubbles and flux ropes on the low- and mid-latitude magnetopause generate bipolar outward/inward (+,-) magnetic field perturbations normal to the nominal magnetopause on both sides of that boundary, while southward-moving bubbles and flux ropes on the low- and mid-latitude magnetopause generate bipolar inward/outward $(-,+)$ perturbations. Spacecraft that enter the events should observe enhanced plasma pressures and craterlike variations in the magnetic field strength when they enter events formed by antiparallel merging, while spacecraft that remain outside should observe transient magnetic field strength enhancements in the magnetic field component normal to the axis of the FTE (Farrugia et al., 1987).

A wide array of observations confirms the predictions of this model. Russell and Elphic (1978) reported observations of flux transfer events marked by transient $(\sim 1 \mathrm{~min})$ magnetic field strength increases and "direct" $(+,-)$ bipolar signatures, while Rijnbeek et al. (1982) reported observations of similar events marked by "reverse" $(-,+)$ bipolar signatures. Subsequent statistical surveys demonstrated that (1) the events are most common on the low-latitude magnetopause during periods of southward IMF orientation, (2) direct signatures predominate on the northern low-latitude magnetopause, but that (3) reverse signatures predominate on the southern low-latitude magnetopause (Rijnbeek et al., 1984; Berchem and Russell, 1984). Taken together, these results confirm that most signatures observed on the 

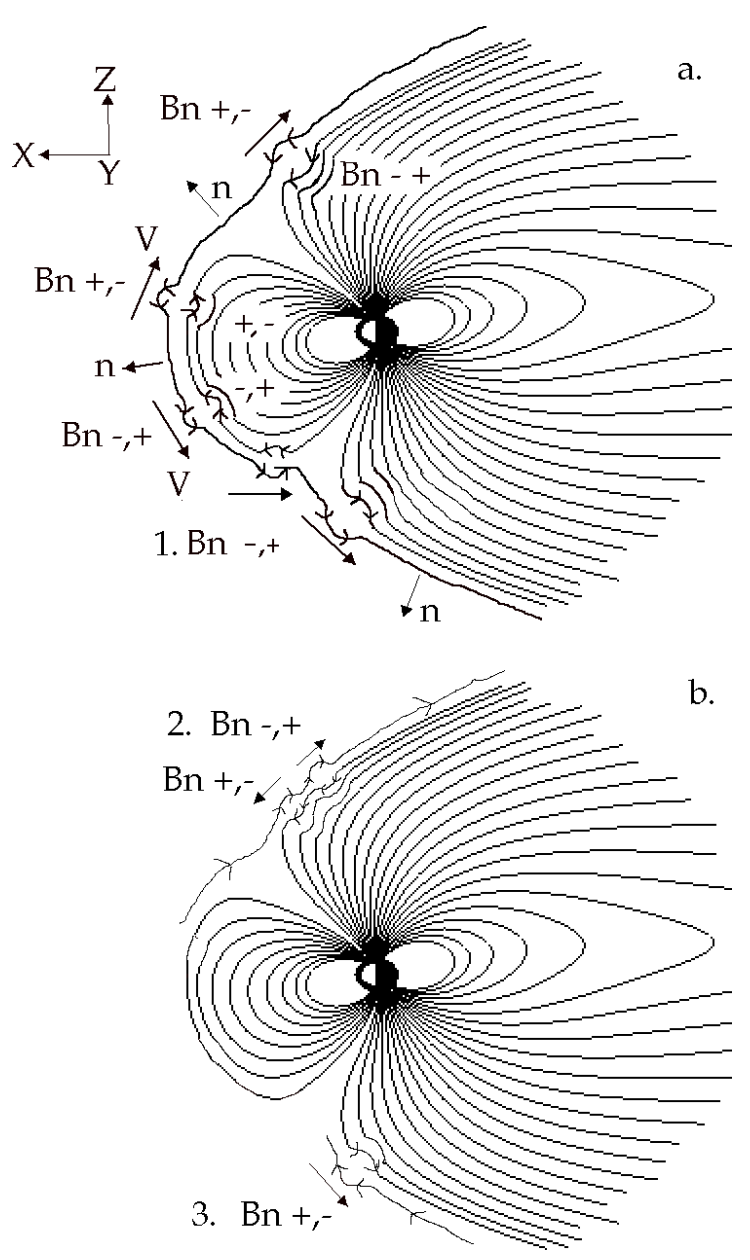

Fig. 1. (a) The bipolar signatures (Bn) normal to the nominal magnetopause predicted for events generated by merging on the subsolar magnetopause during intervals of southward IMF orientation. Direct $(+,-)$ signatures are expected in the magnetosheath north of the merging site, in the dayside equatorial magnetosphere north of the merging site, and in the southern lobe. Reverse $(-,+)$ signatures are expected in the magnetosheath south of the merging site, in the dayside equatorial magnetosphere south of the merging site, and in the northern lobe. The bipolar signatures generated by events in the cusp lie in the z-direction, rather than the direction normal to the nominal magnetopause (position 1). (b) The bipolar signatures normal to the nominal magnetopause predicted for events generated by merging on the high latitude magnetopause during periods of northward IMF orientation. Sunward moving events generate direct signatures on the northern magnetopause, reverse signatures on the southern magnetopause. Antisunward-moving events generate reverse events on the northern magnetopause (position 2), direct events on the southern magnetopause (position 3).

dayside low- and mid-latitude magnetopause correspond to poleward-moving events produced by bursty merging along tilted equatorial merging lines (Russell et al., 1985).

The situation on the high-latitude magnetopause remains considerably less clear. As illustrated in Fig. 1a, events generated by bursty merging on the equatorial magnetopause during periods of southward IMF orientation might move through the cusps to the polar magnetopause. While in the cusps, they should generate bipolar signatures in directions other than that normal to the nominal magnetopause. For example, although the local normal to the nominal magnetopause points in the $+X,-Z$ direction, Event 1 in Fig. 1a generates a $(-,+)$ bipolar signature solely in the $Z$ direction as it moves along the northern edge of the southern cusp in the $-\mathrm{X}$ direction. FTEs that succeed in traversing the cusps should generate bipolar signatures with opposite senses on either side of the high latitude magnetopause: $(+,-)$ signatures in the northern magnetosheath and southern lobe, but $(-,+)$ signatures in the southern magnetosheath and northern lobe.

Alternatively, strongly sheared magnetosheath and magnetospheric magnetic fields may provide the conditions needed for bursty merging and FTE generation on the high-latitude magnetopause (Berchem et al., 1995). Figure 1b illustrates several possibilities. Northward and antisunward magnetosheath magnetic field orientations favor merging on the northern high-latitude magnetopause. The resulting northern hemisphere FTEs generate bipolar $(+,-)$ signatures in both the magnetosheath and the magnetosphere if they move sunward, but $(-,+)$ signatures if they move antisunward (Event 2 in Fig. 1b). Northward and sunward magnetosheath magnetic field orientations favor merging on the southern highlatitude magnetopause. Antisunward moving events generate $(+,-)$ signatures in this location (Event 3 in Fig. 1b).

The first purpose of this paper is to present examples of Interball-1 magnetometer observations illustrating Events 1, 2, and 3 shown in Figs. 1a and b. We will then present a small statistical survey that demonstrates that almost all events move antisunward, confirms that events on the equatorial magnetopause tend to occur for southward magnetosheath magnetic field orientations, but indicates that events on the high-latitude magnetopause are equally likely to occur for northward magnetosheath magnetic field orientations (but more likely to occur for southward IMF orientations). We suggest that events observed on the high-latitude magnetopause can be generated locally during periods of northward IMF orientation, but arrive from the equatorial magnetopause during periods of southward IMF orientation.

\section{Data sets and coordinate systems}

This paper reports the results of a survey of FTEs in Interball-1 observations at the high-latitude dayside magnetopause. Interball-1 was launched on 3 August 1995 into a highly elliptical orbit with apogee $31.4 R_{E}$, inclination $62.8^{\circ}$, and period $92 \mathrm{~h}$ (Zelenyi and Sauvaud, 1997). Observations from the two fluxgate magnetometers, MIF-M (Klimov et al., 1997) and FM-3I (Nozdrachev et al., 1998), on the spacecraft have been intercalibrated and averaged to produce a merged data set with 6-s time resolution that is now available from the CDAWeb data server at the GSFC. To exclude the possibility of any solar wind/magnetosheath trigger for the Interball-1 events, we present Geotail plasma (Mukai et 
28 June 1997

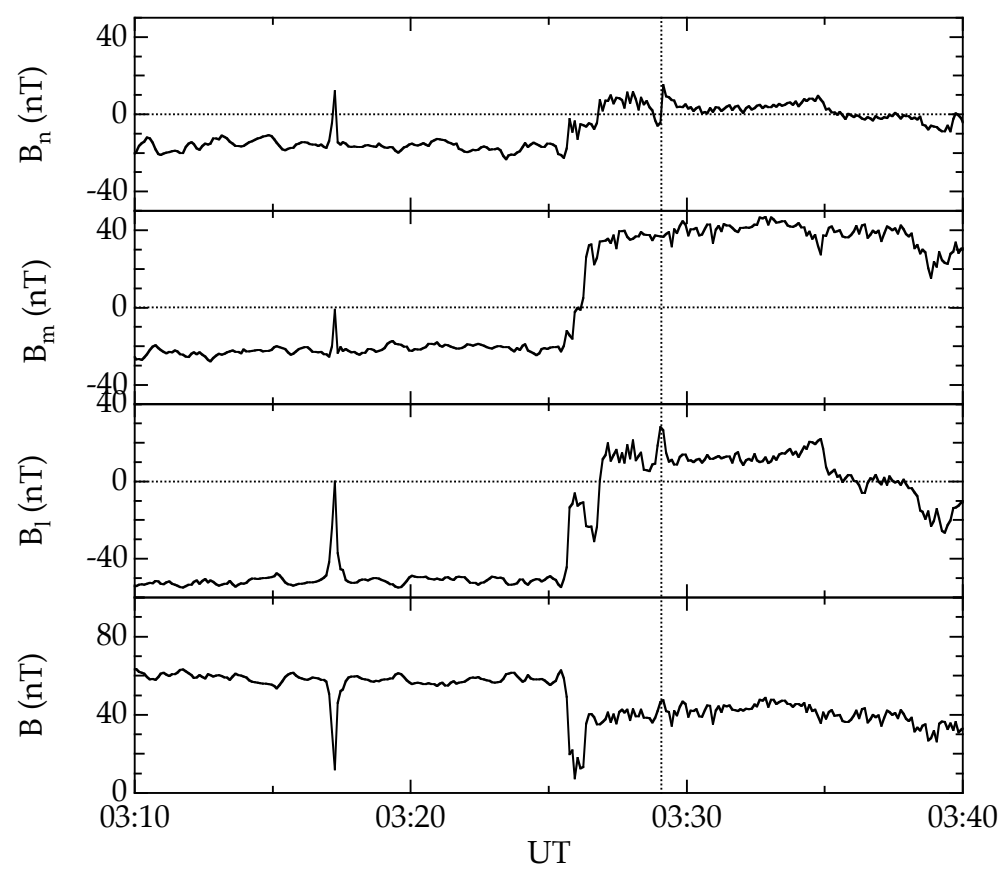

Fig. 2. Interball-1 magnetometer observations from 03:10 to 03:40 UT on 28 June 1997 in boundary normal coordinates (see text). Interball-1 crossed the magnetopause outbound at 03:26 UT. A vertical dashed line identifies a reverse $(-,+) B_{n}$ signature seen in the high-latitude magnetosheath just outside the northern lobe at 03:29 UT.

al., 1994) and magnetic field (Kokubun et al., 1994) observations at 12-s and 3-s time resolution, respectively.

We intentionally employ a variety of coordinate systems. In general, FTEs are most readily identified on the basis of their bipolar signatures normal to the nominal magnetopause. It is therefore often helpful to plot the Interball-1 observations in boundary normal $(\boldsymbol{L} \boldsymbol{M} \boldsymbol{N})$ coordinates (Russell and Elphic, 1978), where $\boldsymbol{N}$ points outward along the local model normal determined from the Roelof and Sibeck (1993) model magnetopause for nominal solar wind conditions (solar wind dynamic pressure $\sim 2 \mathrm{nPa}$, IMF $B_{z} \sim 0$ ), $\boldsymbol{L}$ lies in the plane of the magnetopause and points northward, while $\boldsymbol{M}$ lies in the plane of the magnetopause and points dawnward $(\boldsymbol{M}=\boldsymbol{N} \times \boldsymbol{L})$. However, as noted in the discussion of Fig. 1a, there are occasions when the bipolar signature does not lie in the direction normal to the nominal magnetopause. For these cases, we present the Interball-1 observations in geocentric solar magnetic (GSM) coordinates.

Berchem and Russell (1984) reported that the spatial occurrence patterns of FTEs on the low-latitude dayside magnetopause are best organized in terms of dipole latitude in the solar magnetic (SM) coordinate system. In this coordinate system, the Z-axis lies parallel to the Earth's dipole axis. We will therefore present the spatial occurrence patterns for the Interball-1 events in SM coordinates.

Finally, although no study of FTE occurrence patterns as a function of solar wind conditions has demonstrated that they are best organized when the solar wind observations are presented in GSM coordinates, there is a general expectation that this is the case. Consequently, we present the Geotail interplanetary and magnetosheath magnetic field observations in the GSM coordinate system.

\section{Case study: magnetosheath event on 28 June 1997}

During the interval from $03: 10$ to $03: 40$ UT on 28 June 1997, Interball-1 moved from SM (x, y, z) $=(0.27,-4.02$, $10.81)$ to $(0.75,-4.18,11.22) R_{E}$ in the vicinity of the northern dawn magnetopause. Figure 2 presents Interball-1 magnetometer observations for this interval in $\boldsymbol{L} \boldsymbol{M} \boldsymbol{N}$ coordinates. The spacecraft was initially inside the magnetosphere, where it observed the strong $(\sim 60 \mathrm{nT})$ southward $\left(B_{l}<0\right)$ and duskward $\left(B_{m}<0\right)$ magnetic fields appropriate for its position inside the northern dawn lobe. After a transient encounter at 03:17 UT, the spacecraft entered the magnetosheath proper at 03:26 UT. From 03:26 to 03:35 UT, Interball-1 observed northward and strongly dawnward magnetosheath magnetic fields.

In addition to two brief intervals of very weak magnetic field strengths at 03:17 and 03:26 UT, Interball-1 observed a transient event marked by bipolar $(-,+)$ magnetic field signatures normal to the nominal magnetopause and enhanced magnetic field strengths at 03:29 UT while in the magnetosheath. These are the classic signatures of a reverse FTE. To generate a reverse signature in only the northward component of the northward and dawnward magnetosheath magnetic field, the event must have been moving northward along the magnetopause (e.g., Event 2 in 


\section{June 1997}

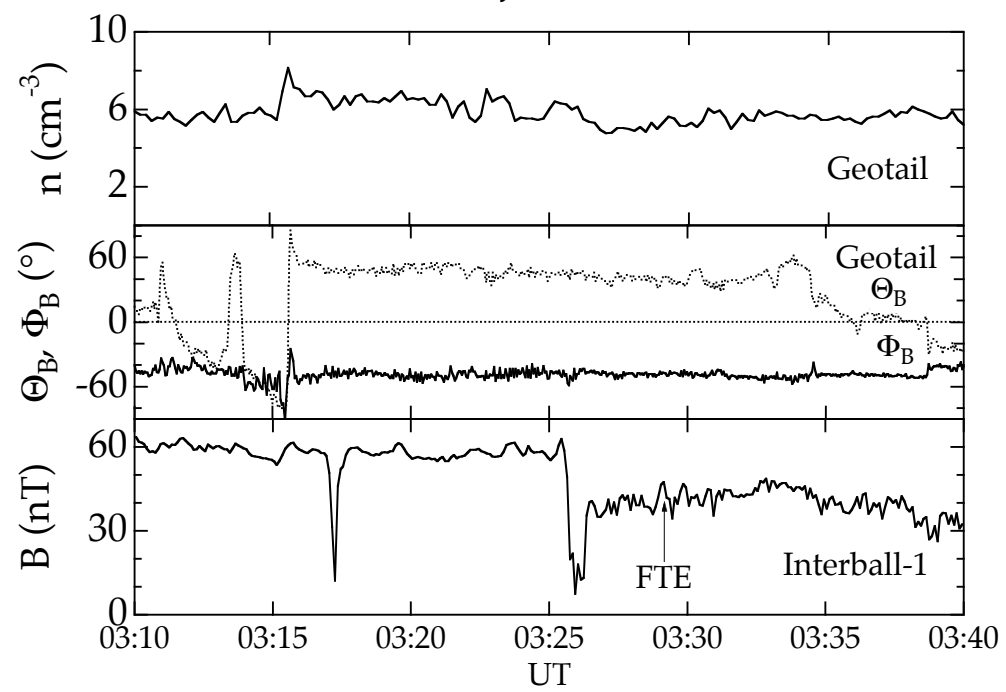

Fig. 3. A comparison of Geotail magnetosheath observations with Interball-1 observations of the magnetopause crossing on 28 June 1997. From top to bottom, the panels in the figure show: Geotail density observations, Geotail magnetic field latitude and longitude observations in GSM coordinates, and Interball-1 magnetic field strength observations.

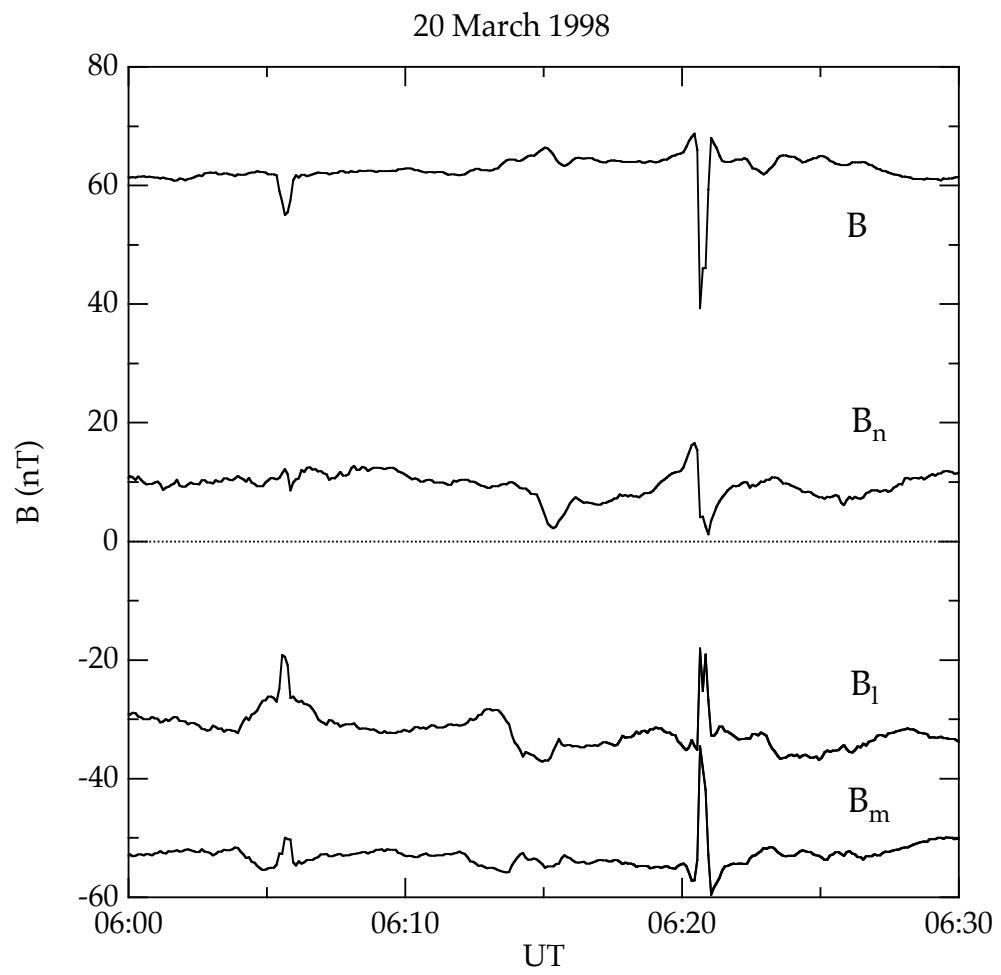

Fig. 4. Interball-1 magnetometer observations from 06:00 to 06:30 UT on 20 March 1998 in boundary normal coordinates. A crater FTE at 06:20 UT follows two less well define events at 06:06 and 0615 UT. All three events occurred within the southern lobe.

Fig. 1b). The strongly sheared magnetosheath and magnetospheric magnetic fields, and the northward component of the magnetosheath magnetic field, suggest an interpretation in terms of local merging on the high latitude magnetopause.
We sought evidence for a solar wind/magnetosheath trigger in Geotail observations. From 03:10 to 03:40 UT, Geotail moved through the dusk magnetosheath from SM $(\mathrm{x}, \mathrm{y}, \mathrm{z})=(0.38,18.25,0.73)$ to $(0.1,18.03,0.34) R_{E}$. 
20 March 1998

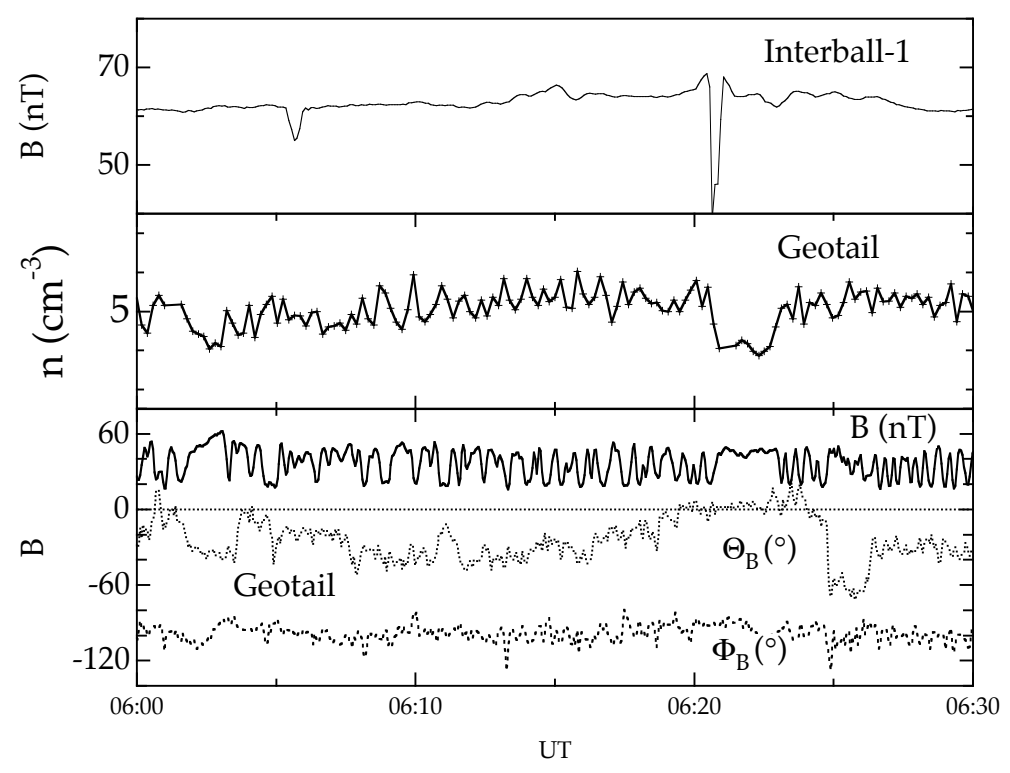

Fig. 5. A comparison of Interball-1 and Geotail observations from 06:00 to 06:30 UT on 20 March 1998. Interball-1 was located in the southern lobe, Geotail in the subsolar magnetosheath. From top to bottom, the figure shows Interball-1 magnetic field strengths, Geotail densities $(n)$, magnetic field strength $(B)$, GSM latitude $(\Theta)$, and GSM longitude $(\Phi)$. Geotail observed a series of drift mirror mode waves marked by antiphase density and magnetic field strength oscillations. If there was any trigger for the crater FTE at Interball-1, it was a density decrease, magnetic field strength increase, and northward magnetosheath magnetic field turning.

Figure 3 presents Geotail density and magnetic field orientations for comparison with Interball-1 magnetic field strength observations during the interval under study. An arrow indicates the time when Interball-1 observed the 03:29 UT FTE. While the sharp reversal from dawnward to duskward magnetosheath magnetic field orientations and attendent density increase may have triggered the 03:16 UT event marked by a weak magnetic fields strength at Geotail, there are no triggers for the weak magnetic field strength seen at the 03:26 UT magnetopause crossing or the 03:29 UT FTE.

\section{Case study: magnetospheric events on 20 March 1998}

From 06:00 to 06:30 UT on 20 March 1998, Interball-1 moved from $\mathrm{SM}=(-0.84,7.85,-8.40)$ to $(-1.08,6.96$, $-8.48) R_{E}$ in the vicinity of the southern dusk magnetopause. Figure 4 presents Interball-1 magnetometer observations for this interval in $\boldsymbol{L} \boldsymbol{M} \boldsymbol{N}$ coordinates. The magnetic field strength was generally high $(>60 \mathrm{nT})$ and the magnetic field pointed southward $\left(B_{l}<0\right)$ and duskward $\left(B_{m}<0\right)$, consistent with expectations for a spacecraft located in the magnetosphere poleward of the southern cusp on the dusk flank.

Interball-1 observed two transient events. The first, at 06:06 UT, exhibited a transient magnetic field strength decrease but no significant bipolar magnetic field signature normal to the nominal magnetopause. The second, from 06:20 to 06:22 UT, was marked by enhanced magnetic field strengths bounding a weak core field, a $(+,-)$ bipolar signature in the component normal to the nominal magnetopause, and a greatly weakened duskward magnetic field component. These are the classic signatures of a direct, crater, FTE. Since the magnetic field pointed southward and duskward, the event is most easily interpreted as an entry into the core region of an FTE moving southward and duskward on the high-latitude southern magnetopause poleward of the cusp (e.g., Event 3 in Fig. 1b).

We sought to identify a magnetosheath trigger for the transient events observed by Interball-1. Geotail moved through the subsolar magnetosheath from SM (x, y, z) $=(9.54$, $-1.74,-2.34) R_{E}$ at $06: 00 \mathrm{UT}$ to $(9.92,-0.96,-2.11) R_{E}$ at 06:30 UT. Figure 5 repeats Interball-1 observations of the magnetospheric magnetic field strength for comparison with Geotail plasma density (12-s time resolution) and magnetic field (3-s time resolution) observations in SM coordinates. The most prominent features in the Geotail observations are a transient decrease in the density and increase in the magnetic field strength from 06:21:30 to 06:23 UT. These perturbations occurred in the midst of a longer interval of slightly northward and strongly dawnward magnetic field orientations from 06:19 to 06:25 UT. During the remainder of the interval shown, Geotail observed large amplitude ( $\delta \mathrm{B} \sim 10 \mathrm{nT}, \delta \mathrm{n} \sim 1 \mathrm{~cm}^{-3}$ ) drift mirror mode waves marked by antiphase density and magnetic field strength variations superimposed upon a dawnward and southward magnetic field. The antisunward magnetosheath velocity (not shown) remained nearly constant at $300 \mathrm{kms}^{-1}$.

It is immediately clear that the variations in the magnetospheric magnetic field strength observed by Interball-1, in 
4 July 1998

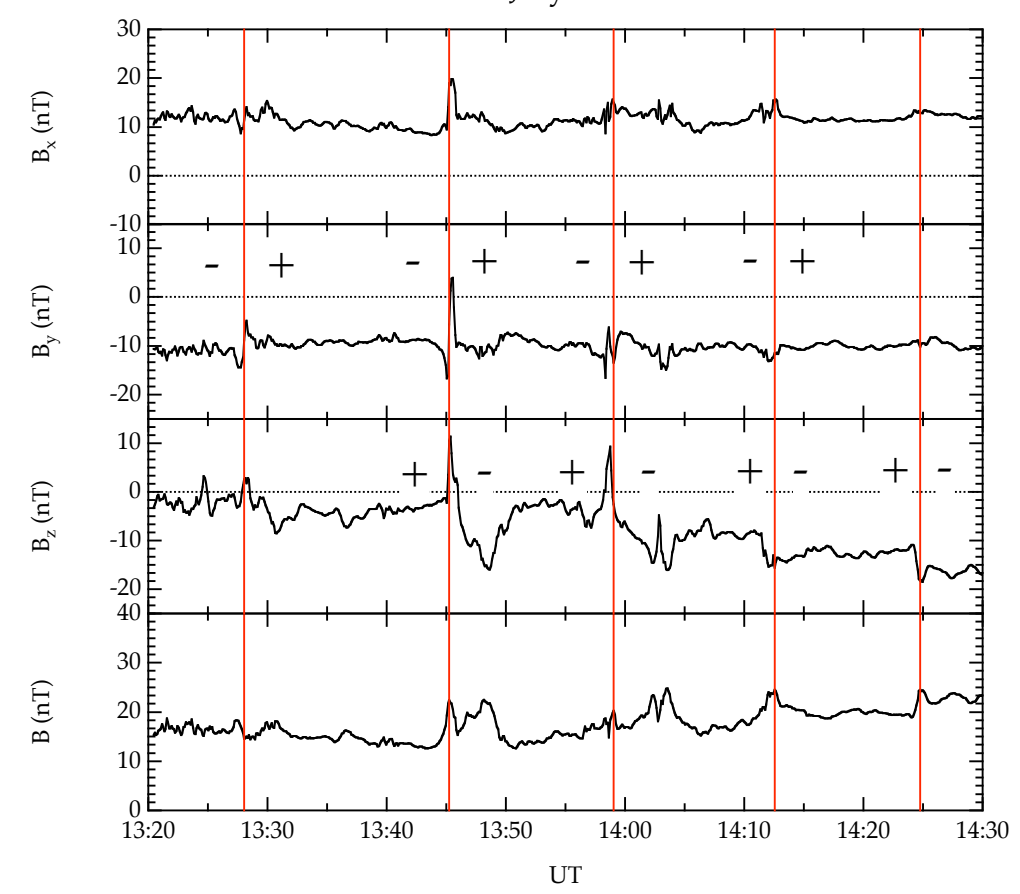

Fig. 6. Interball-1 magnetometer observations from 13:20 to 14:30 UT on 4 July 1998 in GSM coordinates. Interball-1 remained within the magnetosphere throughout the interval, where it observed the sequence of FTEs marked by vertical lines. The bipolar signatures were initially more pronounced in the y-component, but later became more readily visible in the z-component.

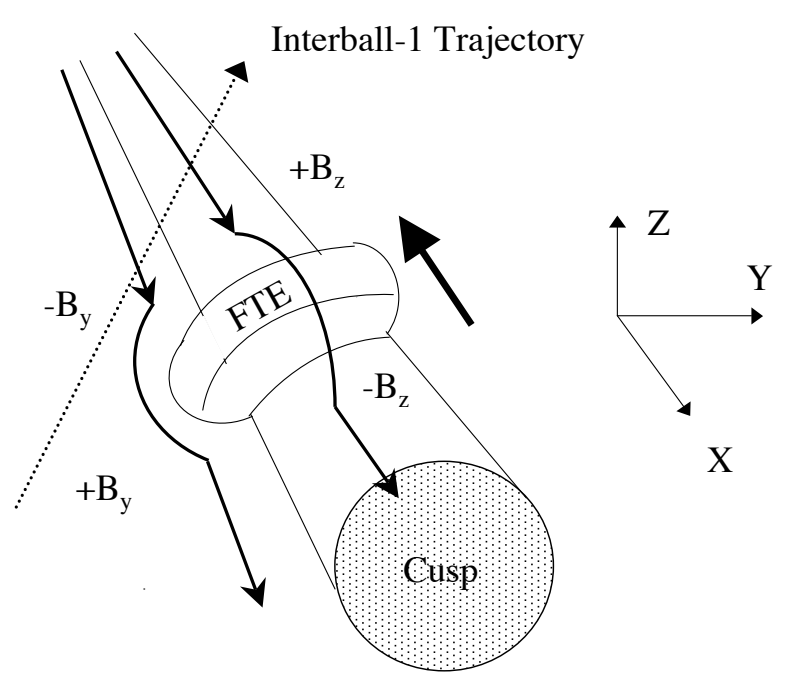

Fig. 7. An interpretation of the Interball-1 observations on 4 July 1998. The southern cusp has finite dimensions in both the y-and $\mathrm{z}$-directions. Field lines point sunward $(+\mathrm{x})$ and diverge in both the $\mathrm{y}$ - and z-directions. An antisunward-moving FTE bulges outward from the cusp into the surrounding regions of magnetospheric magnetic field lines. The passage of this bulge generates bipolar magnetic field signatures in the direction normal to the nominal boundary of the cusp. At locations dawnward of the cusp, the passage of the bulge generates $(-,+) B_{y}$ signatures. At locations northward of the cusp, the passage of the bulge generates (+,-) $B_{Z}$ signatures. particular the transient events at 06:06 and 06:20 UT, did not correspond to the density variations at Geotail. In view of Geotail's subsolar location, we can rule out the possibility that Geotail did not observe the solar wind/magnetosheath input reaching the subsolar magnetopause. Instead, we must interpret the discrepancy in the Geotail and Interball-1 observations as evidence for transient event generation by intrinsic magnetopause processes. In this regard, it is notable that the 06:20 UT FTE occurred in the midst of an interval of slightly northward and strongly dawnward magnetosheath magnetic field orientations. Since Interball-1 observed a southward and strongly duskward magnetospheric magnetic field orientation at this time, conditions were ideal for event generation by a burst of antiparallel merging on the high-latitude magnetopause in the immediate vicinity of Interball-1.

\section{Case study: cusp events on 4 July 1998}

During the interval from 13:20 to 14:30 UT on 4 July 1998, Interball-1 moved from $\mathrm{SM}(\mathrm{x}, \mathrm{y}, \mathrm{z})=(8.46,-4.33,-7.22)$ to $(7.51,-2.79,-7.21) R_{E}$. Figure 6 presents the Interball-1 observations for this interval in the GSM coordinate system, because this is the coordinate system in which they are most easily interpreted. Interball-1 observed nearly constant, strong, positive $12 \mathrm{nT} B_{x}$ and negative $8 \mathrm{nT} B_{y}$ components throughout the interval. The southward $B_{z}$ component gradually increased from $-5 \mathrm{nT}$ to $-18 \mathrm{nT}$, thereby becoming the dominant component by the end of the interval. 


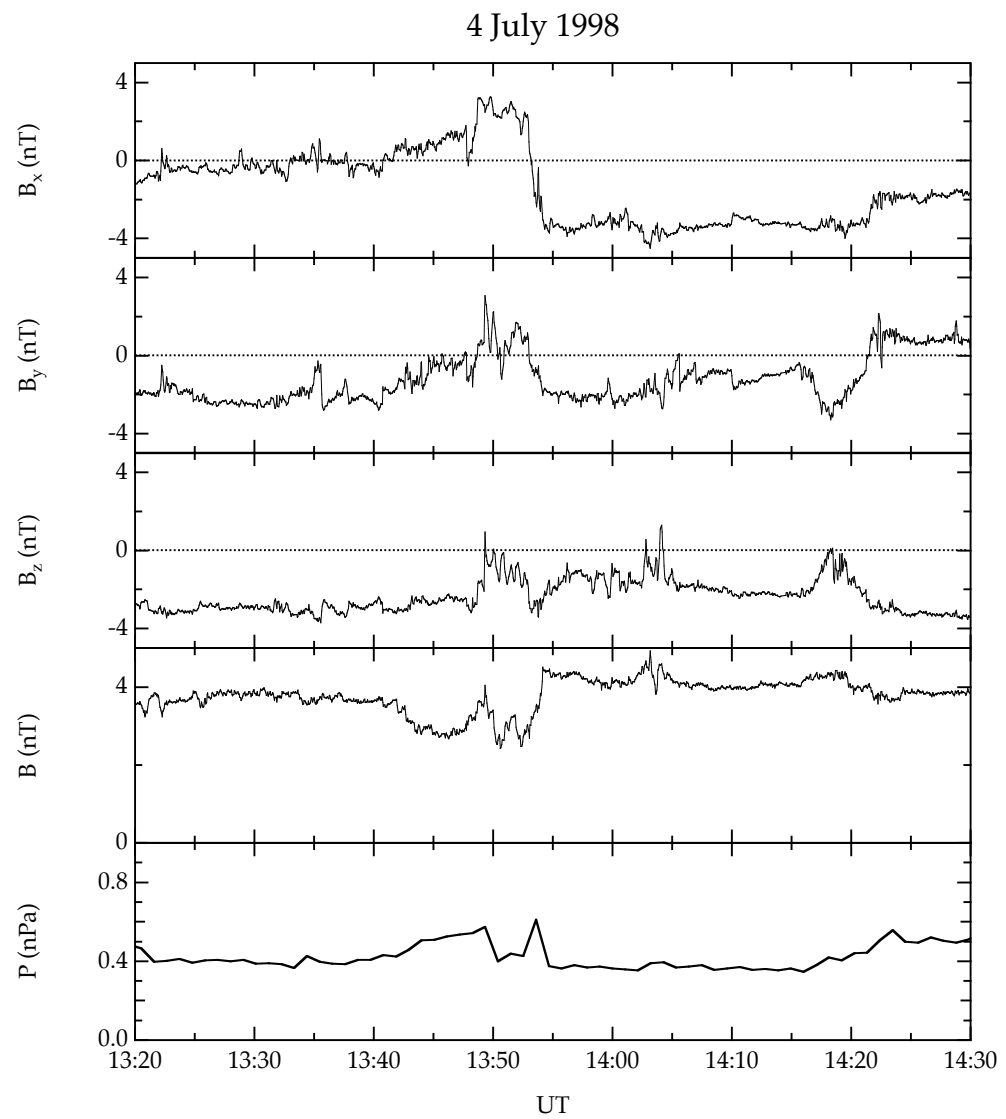

Fig. 8. Geotail dynamic pressure $\left(\mathrm{nMV}^{2}\right)$ and magnetic field observations in GSM coordinates from 13:20 to 14:30 UT on 4 July 1998. Geotail was located in the solar wind, where it generally observed a southward and dawnward IMF orientation. There is no evidence for a sequence of features in either the Geotail plasma or magnetic field observations that could have triggered the events at Interball-1 during the same interval.

Together, the observations are consistent with Interball-1's antisunward and duskward motion through the pre-noon equatorward edge of the southern cusp, a region where sunward, southward, and dawnward magnetic fields are expected. Interball-1 was therefore located in the magnetosphere near position 1 in Fig. 1a.

Vertical lines in Fig. 6 identify 5 transient events in the Interball-1 observations. The average recurrence time was $\sim 14$ min. Bipolar,$-+B_{y}$ signatures mark the events at 13:27 (10 nT), 13:45 (20 nT), 13:59 (10 nT), and 14:12 (only $3 \mathrm{nT}$ ) UT. Bipolar (+,-) $B_{z}$ signatures mark the events at 13:45 (27 nT), 13:59 (23 nT), 14:12 (7nT), and 14:24 $(7 \mathrm{nT})$ UT. Note that the bipolar signatures were not seen in the $B_{x}$ component of the magnetic field, essentially normal to the nominal magnetopause. The time between the peakto-peak bipolar magnetic field signatures exceeded $30 \mathrm{~s}$ for each event. Each event was also accompanied by a magnetic field strength increase.

Figure 7 illustrates an interpretation of the Interball-1 observations. Interball-1 moved antisunward and duskward relative to an idealized cusp. The spacecraft initially lay dawnward of the cusp, but later lay northward of the cusp. A bulge in the cusp dimensions corresponding to an FTE moved antisunward. When the spacecraft lay dawnward from the cusp, the passage of the bulge generated a compression in the magnetic field strength and a $(-,+)$ bipolar signature in the Y-component of the magnetic field. When the spacecraft lay northward from the cusp, the passage of the bulge generated a compression in the magnetic field strength and a (+,-) bipolar signature in the Z-component of the magnetic field. At intermediate locations, the spacecraft observed both signatures.

Bulges in the cusp's dimensions may correspond to flux transfer events or pressure pulse driven waves on the magnetopause. To distinguish between these two possibilities, we must inspect simultaneous solar wind observations. Figure 8 presents Geotail solar wind observations in GSM coordinates for the period from 13:20 to 14:30 UT. During this interval, Geotail moved from GSM (x, y, z) $=(23.5,17.7,-6.6)$ to (23.0, 18.4, -5.8) $R_{E}$ upstream from the dayside bow shock. Geotail observed several tangential discontinuities marked by abrupt rotations in the magnetic field orientation and antiphase density and magnetic field strength variations. The IMF remained southward throughout the interval. Geotail did not observe a sequence of dynamic pressure pulses. 


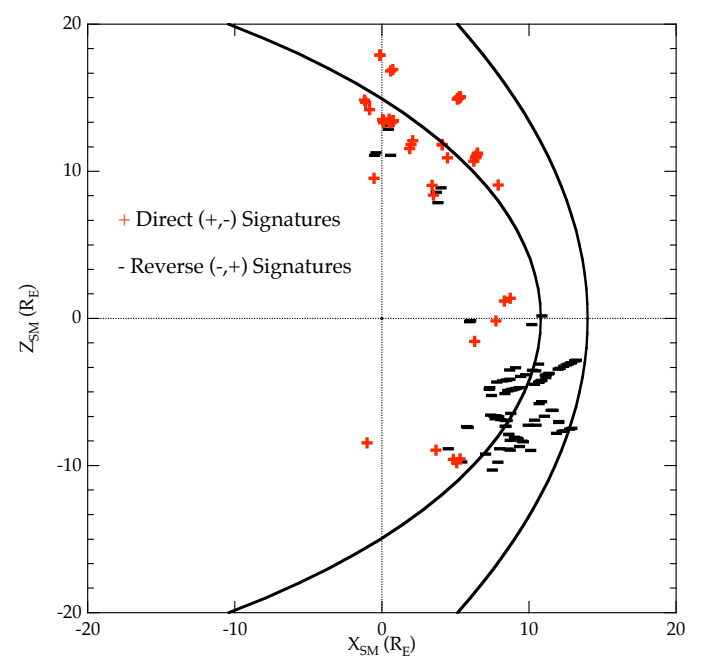

Fig. 9. The locations in the $X-Z$ solar-magnetic (SM) plane of the 162 FTEs that Interball-1 observed from 1995 to 1999 within $10 R_{E}$ of the X-Z plane. Direct events (red crosses) predominate north of the geomagnetic equator, reverse events (black dashes) predominate south of the equator, and a mixture of signatures occur in the vicinity of the geomagnetic equator.

The absence of any relationship between the solar wind variations and the transient events seen by Interball-1 suggests that the Interball-1 events were not caused by antisunward-moving waves driven by solar wind pressure variations or FTEs triggered by fluctuations in the IMF orientation. On the other hand, the Interball-1 events might have been caused by bursts of merging driven by local instabilities on the dayside magnetopause. The southward IMF orientation favored bursty merging on the dayside magnetopause. As illustrated in Fig. 1a, events moving poleward from the posited equatorial merging site and into the southern cusp could have generated the bipolar $(-,+) B_{y}$ and $(+,-) B_{z}$ signatures seen by Interball-1.

\section{Statistical occurrence patterns}

Based on the case studies reported above, past work, and theoretical considerations, one might conclude that events on the equatorial magnetopause tend to occur during periods of southward magnetosheath magnetic field orientation, but that events on the high latitude magnetopause tend to occur during periods of northward magnetosheath magnetic field orientation. To test this hypothesis, we conducted a statistical survey of FTEs observed by Interball-1. We identified FTEs on the basis of (1) a clear symmetric bipolar signature in the component $\left(B_{n}\right)$ of the magnetic field normal to the nominal (Roelof and Sibeck, 1993) magnetopause with peak-to-peak amplitude greater than $4 \mathrm{nT}$, (2) an enhanced or crater-like magnetic field strength, and (3) a peak-to-peak bipolar $B_{n}$ signature duration greater than $30 \mathrm{~s}$. The time interval between events was required to exceed the durations for individual events. We excluded events centered on
FTE Occurrence Patterns

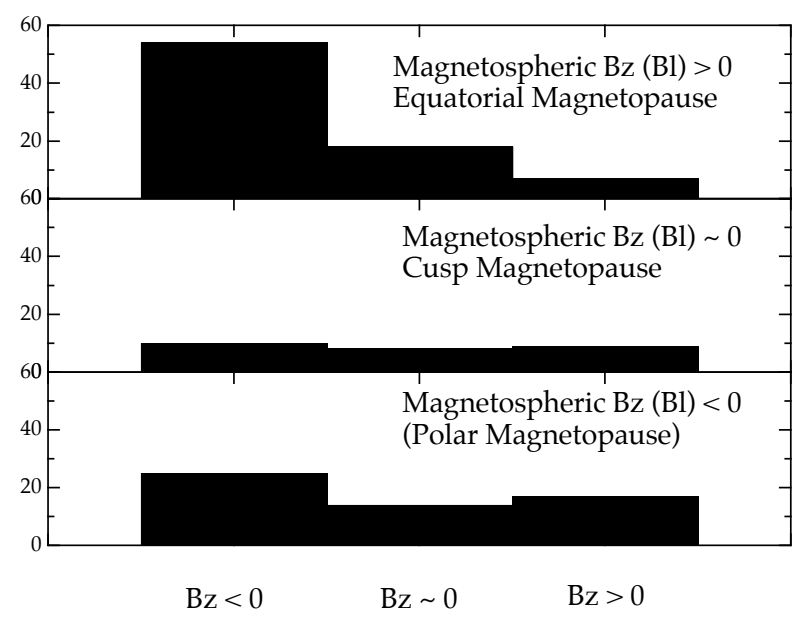

Sheath Field Orientation

Fig. 10. Results of a statistical study of event occurrence versus magnetosheath magnetic field orientation at three locations on the magnetopause. Events on the equatorial magnetopause exhibit a strong tendency to occur for southward magnetosheath magnetic field orientations. Events in the cusp show no tendency to occur for northward or southward magnetosheath magnetic field orientations. Events on the polar magnetopause show a weak tendency to occur for both strongly northward and southward magnetosheath magnetic field orientations.

magnetopause crossings and those in which the bipolar signature was not centered on magnetic field strength increases.

Figure 9 shows the locations of the 162 FTEs that Interball-1 observed from 1995 to 1999 within $10 R_{E}$ of the $\mathrm{X}-\mathrm{Z}$ solar-magnetic (SM) plane projected into that plane. As expected on the basis of past work, direct events (red crosses) predominate north of the geomagnetic equator, reverse events (black dashes) predominate south of the equator, and a mixture of signatures occurs in the vicinity of the geomagnetic equator. Nevertheless, there are exceptions. Several reverse events occurred on the antisunward edge of the distribution at northern latitudes (including the event on 28 June 1997), while several direct events occurred on the antisunward edge of the distribution at southern latitudes (including the events on 20 March 1998).

We subdivided the 162 events whose positions are shown in Fig. 9 into three categories: those occurring equatorward of the cusp (northward magnetospheric magnetic field just inside the nearest magnetopause crossing), those occurring poleward of the cusp (southward magnetospheric magnetic field just inside the nearest magnetopause crossing), and those occurring in or near the cusp $\left(B_{l}<10 \mathrm{nT}\right.$ just inside the nearest magnetopause crossing).

For events occurring within the magnetosphere, we took the magnetospheric magnetic field orientation as that at the time of the event, and the magnetosheath magnetic field orientation as that immediately outside the nearest magnetopause crossing. There were 89 events for southward 
FTE Occurrence Patterns

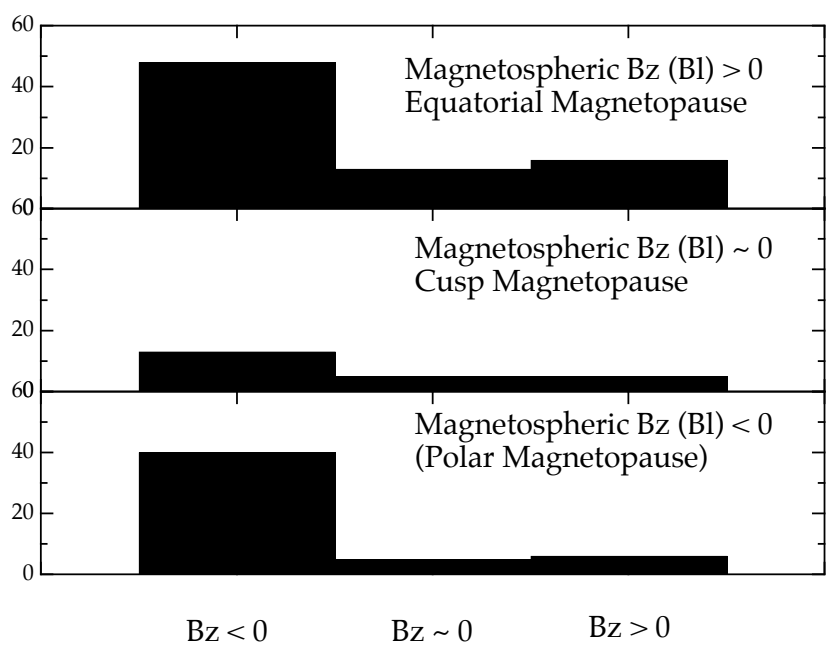

IMF Orientation

Fig. 11. Results of a statistical survey of event occurrence versus IMF orientations at three locations on the magnetopause. Events on all regions of the magnetopause exhibit a strong tendency to occur for southward IMF orientations.

magnetosheath magnetic field orientations, 40 for weak fields or ecliptic orientations, and 33 for northward sheath magnetic field orientations. There were 79 events on the equatorial magnetopause, 56 on the high-latitude magnetopause, and 27 near the cusp.

Figure 10 confirms previous work demonstrating that events on the dayside equatorial magnetopause tend to occur for southward magnetosheath magnetic field orientations. However, in contrast to the hypothesis that events on the high-latitude magnetopause occur predominantly for northward magnetosheath magnetic field orientations, Fig. 10 indicates that they are equally likely for northward and southward orientations. We also find no tendency for events in the cusp to occur for northward or southward magnetosheath magnetic field orientations.

One possible interpretation of these results is that some or all the events observed at the high-latitude magnetopause were actually generated on the equatorial magnetopause for southward magnetosheath magnetic field orientations but then moved poleward into regions where the draped magnetosheath magnetic field pointed northward. Coleman (2005) surveyed Interball-1 and Geotail magnetosheath magnetic field orientations just outside the subsolar, flank, and highlatitude magnetopause as a function of IMF orientation. They found that subsolar magnetosheath magnetic field orientations resemble those in the solar wind, that those at highlatitude often differ, and that those on the magnetotail flanks often differ substantially.

Consequently, we surveyed Wind, Geotail, and IMP-8 IMF observations for each of the 162 events in our data base. To reduce errors in estimating lag times from the IMF monitor to the magnetopause, we preferentially chose to use

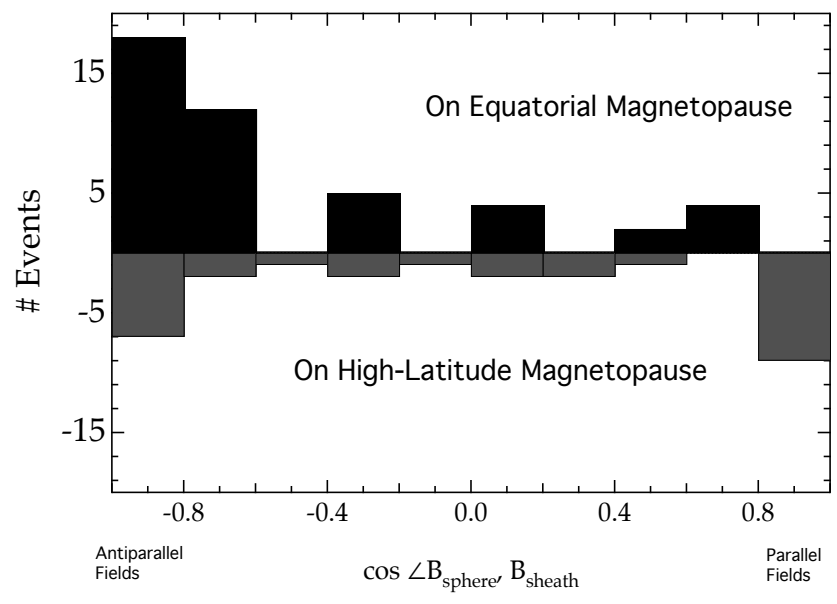

Fig. 12. Event occurrence versus the cosine of the shear angle between the magnetosheath and magnetospheric magnetic fields. Events on the equatorial magnetopause show a strong tendency to occur for high shears, while those on the high-latitude magnetopause exhibit a marked tendency to occur for both strongly and weakly sheared magnetic fields.

Geotail or IMP-8 observations. When both Geotail and IMP8 were available, we used observations from the spacecraft nearest the Sun-Earth line. For simplicity, we calculated arrival times under an assumption that the solar wind discontinuities lay perpendicular to the Sun-Earth and were advected anti-sunward with the solar wind velocity. Several events corresponding to abrupt changes in the IMF $B_{z}$ component are excluded from the study, while events occurring for IMF $\left|B_{z}\right|<1 \mathrm{nT}$ are assigned to the IMF $B_{z} \sim 0$ category. Figure 11 presents the results of this survey. Now it becomes clear that most events on the equatorial, cusp, and polar magnetopause occurred for southward IMF orientations. Consequently, it is very likely that some events observed on the high-latitude magnetopause for northward magnetosheath magnetic field orientations were actually generated on the equatorial magnetopause where southward IMF orientations favor reconnection.

Nevertheless, some events observed at the high-latitude magnetopause occur for both northward IMF and magnetosheath magnetic field orientations. It is easiest to interpret these events in terms of bursty antiparallel merging on the high-latitude magnetopause. The magnetospheric magnetic field poleward of the cusp often has a large dawn/dusk component. To better test the predictions of the antiparallel merging model, we sought to determine the angle between the magnetosheath and magnetospheric magnetic field for each of the events in our data base. To reduce the chance of the magnetic field orientation at the magnetopause changing during the time interval between magnetopause crossing and event observation, we excluded events that occurred more than $30 \mathrm{~min}$ from the nearest magnetopause crossing, leaving a data base of only 72 events. 


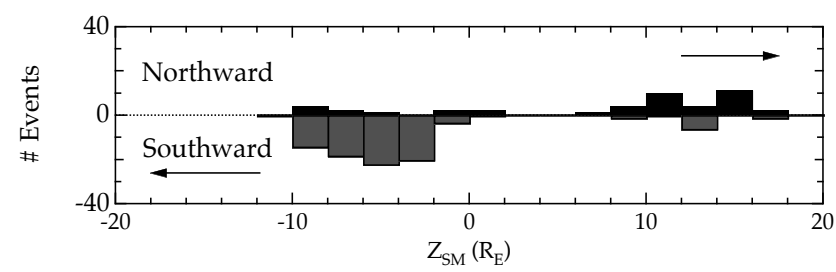

Fig. 13. The direction of motion in which the events move as inferred from the sense of their bipolar signatures and the background magnetic field. Almost all events observed south of the geomagnetic equator $(\mathrm{Z}=0)$ move southward, while almost all events observed north of the geomagnetic equator move northward.

Figure 12 shows that events on the equatorial magnetopause exhibit a strong tendency to occur for antiparallel magnetosheath and magnetospheric magnetic fields, consistent with the predictions of the antiparallel merging model and past surveys (Berchem and Russell, 1984; Rijnbeek et al., 1984). By contrast, the figure shows that events on the high-latitude magnetopause tend to occur for both strongly parallel and antiparallel magnetosheath and magnetospheric magnetic fields. Note, however, that events on the highlatitude magnetopause rarely occur for perpendicular magnetosheath and magnetospheric magnetic fields. The simplest interpretation of these results is to invoke two sources for events on the high-latitude magnetopause: local reconnection during periods when the magnetosheath and magnetospheric magnetic fields lie nearly antiparallel (Fig. 1b), and the equatorial magnetopause when local magnetosheath and magnetospheric magnetic fields lie nearly parallel (Fig. 1a).

If so, the magnetosheath flow must sweep equatorial events poleward past the cusps to locations on the highlatitude magnetopause. We have used the observed magnetosheath/magnetosphere magnetic field orientation and the sense of the bipolar magnetic field signature normal to the nominal magnetopause to determine the direction of motion of the 137 events in our data base for which $B_{z}$ was significantly non-zero as a function of distance from the SM equatorial plane. As can be seen in Fig. 13, almost all of the events that Interball-1 observed north of the geomagnetic equator moved northward, whereas almost all of the events the spacecraft observed south of the equator moved southward. Apparently, the magnetosheath flow sweeps events generated locally on the high-latitude magnetopause, and those generated on the equatorial magnetopause, antisunward.

\section{Conclusions}

We presented three case studies of flux transfer events on the high-latitude magnetopause. Two of the case studies demonstrated that events observed just inside and outside the magnetopause at latitudes poleward of the cusp can occur for strongly northward magnetosheath magnetic field orientations. We attributed these events to local, antiparallel, reconnection on the high-latitude magnetopause. The third case study provided examples of FTEs observed in the vicinity of the cusp. They were identified on the basis of bipolar signatures perpendicular to the cusp boundaries rather than the nominal magnetopause. We interpreted these events, which occurred during an interval of southward IMF orientation, as evidence for the poleward motion of FTEs generated on the equatorial magnetopause into the cusps. Because we were unable to identify any solar wind trigger for any of the events studied in this paper, we attributed all of them to bursty merging triggered by local instabilities on the magnetopause rather than north/south IMF turnings or solar wind pressure pulses.

This is not the first paper to present observations of FTEs on the high-latitude magnetopause. Berchem et al. (1995) reported a case study of FTEs observed by Hawkeye-1 on the high-latitude northern magnetopause near local midnight, also during an interval of northward magnetosheath magnetic field orientations. Three events (each of duration $\sim 1 \mathrm{~min}$ ) were marked by near-oscillatory $(T \sim 1 \mathrm{~min})$ magnetic field signatures normal to the nominal magnetopause, while the fourth exhibited a monopolar spike normal to the nominal magnetopause. There was no magnetic field strength increase for any of the four events. Sonnerup et al. (2004) reported Cluster observations of an FTE observed in the magnetosheath just after local noon and just equatorward of the cusp during an interval when the magnetosheath magnetic field pointed strongly dawnward, but neither northward or southward. The event exhibited near monopolar signatures in the $\mathrm{X}$ and $\mathrm{Z}$ components of the magnetic field. Finally, Thompson et al. (2004) interpreted Cluster observations of a series of transient events that occurred poleward of the northern cusp just prior to local noon as evidence for FTEs. A reversal in $B_{z}$, but no magnetic field strength increase or bipolar signature normal to the nominal magnetopause, marked each event. As they are not classic examples of FTEs, we did not include events of the type reported by Berchem et al. (1995), Sonnerup et al. (2004), or Thompson et al. (2004) in our study.

We considered the properties of the dayside events observed by Interball- 1 on a statistical basis. Consistent with previous studies, we found that events observed on the equatorial and mid-latitude magnetopause equatorward of the cusps exhibit a strong tendency to occur for southward magnetosheath magnetic field orientations. By contrast, events observed on the high-latitude magnetopause, poleward of the cusps, are equally likely to occur for northward and southward magnetosheath magnetic field orientations. However, they exhibit a marked tendency to occur for nearly parallel or antiparallel magnetosheath and magnetospheric magnetic field orientations. Furthermore, many of the events observed on the high-latitude magnetopause during intervals of northward magnetosheath magnetic field orientation actually occurred during intervals of southward IMF orientation. We interpreted this result as evidence indicating that events on the high-latitude magnetopause are generated both 
locally (during periods of northward IMF) and on the equatorial magnetopause (during periods of southward IMF).

A survey of event motion as a function of distance from the equator confirmed this suggestion. Almost all events observed north of the equator move northward, while almost all those observed south of the equator move southward. Although bursts of merging should produce pairs of bubbles that move both poleward and equatorward away from merging lines, our results suggest that these events are rapidly entrained by the ambient magnetosheath flow and swept antisunward past the high-latitude magnetopause. Consistent with this, the numerical simulations of Ku and Sibeck (2000) indicate that the velocities of FTEs propagating opposite the magnetosheath flow diminish relative to those propagating in the direction of the magnetosheath flow.

Acknowledgements. We thank both referees for helpful comments that significantly improved both the interpretation and presentation. Geotail plasma and magnetic field observations were provided by the DARTS WWW server. NASA GSFC's CDAWeb services were used to survey Wind, IMP-8, and Geotail IMF observations. Interball-1 ELECTRON observations, kindly supplied by J.-A. Sauvaud, were used to help identify magnetopause crossings. This research was supported by NASA's SEC Guest Investigator program. In particular, research at the University of Maryland was supported by NASA Grant NNG05GJ16G.

Topical Editor T. Pulkkinen thanks Y. Wang and another referee for their help in evaluating this paper.

\section{References}

Berchem, J. and Russell, C. T.: Flux transfer events on the magnetopause: Spatial distribution and controlling factors, J. Geophys. Res., 89, 6689-6703, 1984.

Berchem, J., Raeder, J., and Ashour-Abdalla, M.: Reconnection at the magnetospheric boundary: Results from global magnetohydrodynamic simulations, in: Physics of the Magnetopause, edited by: Song, P., Sonnerup, B. U. Ö., and Thomsen, M. F., AGU, Washington DC, 205-213, 1995.

Coleman, I. J.: A multi-spacecraft survey of magnetic field draping in the dayside magnetosheath, Ann. Geophys., 23, 885-900, 2005 ,

SRef-ID: 1432-0576/ag/2005-23-885.

Cowley, S. W. H.: The causes of convection in the Earth's magnetosphere: A review of developments during the IMS, Rev. Geophys. Space Phys., 20, 531-565, 1982.

Cowley, S. W. H. and Owen, C. J.: A simple illustrative model of open flux tube motion over the dayside magnetopause, Planet. Space Sci., 37, 1461-1475, 1989.
Farrugia, C. J., Elphic, R. C., Southwood, D. J., and Cowley, S. W. H.: Field and flow perturbations outside the reconnected field line region in flux transfer events: Theory, Planet. Space Sci., 35, 227-240, 1987.

Klimov, S., Romanov, S., Amata, E., et al.: ASPI experiment: Measurement of fields and waves onboard the INTERBALL-1 spacecraft, Ann. Geophys., 15, 514-527, 1997,

SRef-ID: 1432-0576/ag/1997-15-514.

Kokubun, S., Yamamoto, T., Acuña, M., Hayashi, K., Shiokawa, K., and Kawano, H.: The Geotail magnetic field experiment, J. Geomagn. Geoelectr., 46, 7-21, 1994.

$\mathrm{Ku}$, H.-C. and Sibeck, D. G.: Flux transfer events produced by the onset of merging at multiple X lines, J. Geophys. Res., 105, 2657-2676, 2000.

Lee, L.-C. and Fu, Z.-F.: A theory of magnetic flux transfer at the Earth's magnetopause, Geophys. Res. Lett., 12, 105, 1985.

Mukai, T., Machida, S., Saito, Y., Hirahawa, M., Terasawa, T., Kaya, N., Obara, T., Ejiri, M., and Nishida, A.: The low-energy particle (LEP) experiment onboard the Geotail satellite, J. Geomag. Geoelec., 46, 669-692, 1994.

Nozdrachev, M. N., Skalsky, A. A., Styazhkin, V. A., and Petrov, V. G.: Some results of measurements by the FM-3I flux-gate instrument onboard the Interball-1 spacecraft, Cosmic Res., 36, 251, 1998.

Rijnbeek, R. P., Cowley, S. W. H., Southwood, D. J., and Russell, C. T.: Observations of "reverse polarity" flux transfer events at the Earth's dayside magnetopause, Nature, 300, 23-26, 1982.

Rijnbeek, R. P., Cowley, S. W. H., Southwood, D. J., and Russell, C. T.: A survey of dayside flux transfer events observed by ISEE 1 and 2 magnetometer, J. Geophys. Res., 89, 786-800, 1984.

Roelof, E. and Sibeck, D. G.: Magnetopause shape as a bivariate function of interplanetary magnetic field $\mathrm{Bz}$ and solar wind dynamic pressure, J. Geophys. Res., 98, 21 421-21 450, 1993.

Russell, C. T. and Elphic, R. C.: Initial ISEE magnetometer results: Magnetopause observations, Space Sci. Rev., 22, 681-715, 1978

Russell, C. T., Berchem, J., and Luhmann, J. G.: On the source regions of flux transfer events, Adv. Space Res., 5, 363-368, 1985.

Sonnerup, B. U. Ö., Hasegawa, H., and Paschmann, G.: Anatomy of a flux transfer event seen by Cluster, Geophys. Res. Lett., 31, doi:10.1029/2004GL020134, 2004.

Southwood, D. J., Farrugia, C. J., and Saunders, M. A.: What are flux transfer events?, Planet. Space Sci., 36, 503-508, 1988.

Thompson, S. M., Kivelson, M. G., Khurana, K. K., Balogh, A., Reme, H., Fazarkeley, A. N., and Kistler, L. M.: Cluster observations of quasi-periodic impulsive signatures in the dayside northern lobe: High-latitude flux transfer events?, J. Geophys. Res., 109, A02213, doi:10.1029/2003JA010138, 2004.

Zelenyi, L. M. and Sauvaud, J.-A.: Special Topic Interball-1: First scientific results, Ann. Geophys., 15, 511-513, 1997,

SRef-ID: 1432-0576/ag/1997-15-511. 